\title{
How Can Sharing Leadership Stimulate Employee Innovative Behavior: On the Role of Innovation Self- Efficacy and Emotional Commitment
}

\author{
Li Hui ${ }^{1}$
}

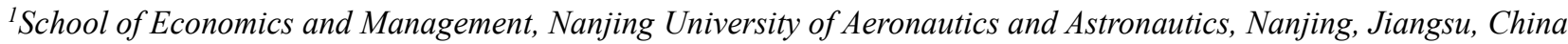
lihuihit666@163.com
\end{abstract}

\begin{abstract}
Exploring the influence mechanism of sharing leadership on employees' innovative behavior is a new perspective to understand the problem of "how to stimulate employees' innovative behavior". Based on social exchange theory and self-recognition theory, this paper introduces innovative self-efficacy as a mediating variable and emotional commitment as a moderating variable in the relationship between sharing leadership and employee innovation behavior, and constructs a moderated mediation model. Through the effective sample data of 188 employees from high-tech enterprises, the theoretical hypothesis model is empirically tested. The research results show that sharing leadership not only has a direct positive impact on employee innovation behavior, but also significantly affects employee innovation behavior through the mediating effect of innovative self-efficacy; emotional commitment positively regulates the relationship between sharing leadership and employee innovation behavior; The positive moderating effect of emotions commitment on sharing leadership and employees' innovative behavior is brought into play through the mediating role of innovative self-efficacy.
\end{abstract}

Keywords: Sharing leadership, innovation self-efficacy, emotional commitment, employee innovative behavior

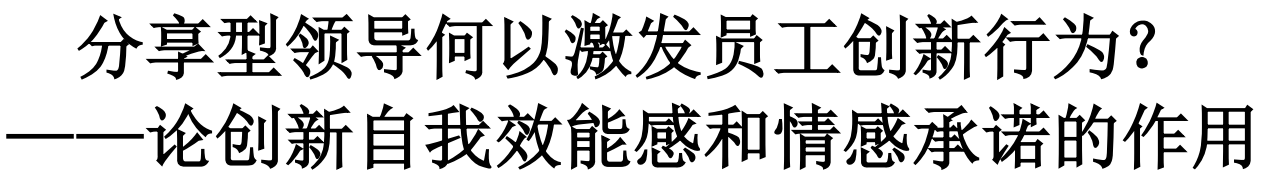

\begin{abstract}
李辉 1
${ }^{1}$ 南京航空航天大学经济与管理学院, 南京, 江苏, 中国 lihuihit666@163.com

\section{摘要}

探究分享型领导对于员工创新行为的影响机制是理解“如何激发员工创新行为”问题的新视角。以社会交换理论 和自我角色认同理论为基础, 在分享型领导与员工创新行为的关系中引入创新自我效能感作为中介变量, 情感 承诺作为调节变量, 构建了一个有调节的中介模型。通过对 188 份来自高新技术企业的员工的有效样本数据, 实证检验了理论假设模型。研究结果表明：分享型领导不仅仅对员工创新行为有直接正向影响，还通过创新自 我效能感的中介作用显著影响员工创新行为; 情感承诺正向调节分享型领导与员工创新行为的关系; 情感承诺 对分享型领导与员工创新行为的正向调节作用通过创新自我效能感中介作用得以发挥。

\section{关键词：分享型领导，创新自我效能感，情感承诺，员工创新行为}

\section{1.前言}

“十四五”是我国创新驱动发展，实现全面塑造发 展新优势的关键五年，为实现我国经济的稳中有进，

就需要在挑战中，不断的发掘创新点，培育创新新动 能, 开辟新的发展局面。面对充满变数的生存环境, 企业需要进行比以往更多的创新活动, 在高风险, 高 难度的创新活动中，企业内各种资源的协调和整合，
\end{abstract}


都需要优秀的企业领导人的谋划和组织。

新的领导力概念不断被学者们提出和研究, 如包 容型领导 ${ }^{[1]}$, 自我牺牲型领导 ${ }^{[2]}$ 和分享型领导 ${ }^{[3,4]}$ 等。 如何激发员工产生新的创新想法, 并将创新想法落实 为具体的创新产品, 如何构建和谐的领导一员工关系 以实现持续的创新 ${ }^{[5]}$, 成为现今许多企业领导者面临 的关键问题。纵观现有的领导力研究, 可以看到新的 领导行为倾向于去中心化、共享和价值生态，这种新 的研究趋势更关注于企业领导者的自我超越, 以“分 享、舍得”的视角 ${ }^{[6]}$ 建立新型、和谐的领导一员工关系。 同样可以看到, 越来越多的心理学概念不断向管理学 领域迁移, 如积极情绪, 内在工作动机, 基于优势的 心理氛围 ${ }^{[7]}$ 等, 跨领域、跨学科间理论的交流愈发密 切, 这使得对于员工创新行为的研究视角更加开阔, 研究也更具新颖性。

纵观已有文献, 发现关于分享型领导和员工创新 行为两者关系的研究仍然存在不足。第一、分享型领 导作为一种新颖的领导方式, 相关研究仍处于理论探 索阶段 ${ }^{[3,4]}$, 其后果变量、作用机制等实证研究较为缺 之。在原有领导力的研究当中, 学者们在领导特质、 行为、权变能力、领导一员工关系、行为绩效等方面 进行了大量的研究, 这些研究倾向于领导和员工互动 关系的态度、情绪、权威等影响过程 ${ }^{[3,4]}$, 却忽视了对 于员工在物质、资源等层面的激励过程, 这显然不利 于全面了解领导实践行为。分享型领导力的新提出, 虽然弥补了这一不足, 但其后果变量的研究少之又少。 第二、关于分享型领导对员工创新行为的影响机理, 现有研究还未曾对其有清晰的阐释和实证支持。鉴于 此, 本文将创新自我效能感和情感承诺这两个变量, 纳入分享型领导影响员工创新行为的整体分析框架, 尝试通过实证检验, 揭开分享型领导的作用“黑箱”。 希望能够拓展分享型领导的后果变量的研究, 也希望 能够在企业实践方面, 为领导充分发挥分享型领导力 以激发员工创新行为提供新的理论参考。

\section{2.文献回顾与研究假设}

\section{1. 分享型领导与员工创新行为}

分享型领导是领导者主动与下属员工分享资源、 福利、利益并积极创造机会让员工参与企业决策过程 的一种领导方式 ${ }^{[3]}$, 分为利益分享和决策分享。分享 型领导关注的重点在于领导过程中利益和决策权的 分享问题。分享型领导是一种介于垂直性领导和分布 式领导的领导方式, 对于领导权力和影响力的适中搭 配，有利于缓解“家长式的权威”，并提升下属员工的 创造性。龙立荣和陈琇霖则从企业和谐角度认为, 分 享型领导指领导主动将个人应得的资源、发展成果与 员工互惠分享, 以实现企业和谐的行为表现 ${ }^{[4]}$ 。主要 包含名利分享和权力分享。名利分享指的是领导者将 自身的一部分经济或名誉利益让渡给下属员工; 权力 分享指的是常见的决策参与也含有一些能降低权力 距离的特权 (如专权使用的设施)。
员工创新行为指“员工在工作过程中，产生创新 构想或问题解决方案, 并将其付予实践”的行为。创新 过程可分为创意产生和创意执行两个阶段 ${ }^{[9]}$ 。前者是 探索新知识, 产生新想法的过程; 后者是将创意落实 为结果的过程。创意从产生到落地实现的整个过程, 需要大量的资源和权力支持。社会交换理论认为, 人 的行为是一种理性的行为, 人们之间的交互活动, 是 为了追求个人利益, 领导和员工之间也同样存在着某 种交换关系。利益分享意味着领导者将资源、利益等 与员工分享，这种行为的本身，基于一种“舍即是得” 的理念 ${ }^{[6]}$, 即让渡自身利益, 使得下属员工受益时, 能够基于互惠原则 $[10]$, 产生更有价值的行为。领导进 行合理的利益分享能够提升员工的获得感和组织支 持感 ${ }^{[11]}$, 能够促进员工创造力的提升。权力分享指领 导者积极向员工提供参与企业决策的氛围和机会, 是 一种鼓励员工建言献策的领导方式, 其本质是一种授 权行为, 通过管理权力的授权, 能够赋予员工较多的 自主权力, 具备开拓新想法和容纳创新风险的空间, 使得员工的创新潜力在低顾虑的环境下更好的转化 为创新实践 ${ }^{[12]}$ 。在权力分享的同时, 员工也得到了心 理授权 ${ }^{[13]}$, 积极心理学许多研究表明, 得到尊重、信 任员工能够提升创新的内部动机 ${ }^{[14,15]}$, 积极影响员工 的创新行为。因此我们提出研究假设 $\mathrm{H} 1$ :

H1: 分享型领导对员工创新行为具有正向影响。

\section{2. 创新自我效能感的中介作用}

创新自我效能感是个体对自己能够在特定任务 或工作中表现创新行为的能力和信心的评价 ${ }^{[16]}$ 。其实 质在于个体创造性达成创新目标的信念, 兼顾创新行 为的过程和结果 ${ }^{[9]}$ 。该概念最初源于 Bandura 所提出 的“自我效能感”，意为“个体对自己达成某项任务或 工作行为能力的信念”, 该概念强调从个体觉知视角 出发的主观感受, 而非个体具有的能力本身。自我效 能感与人们的思维、动机、行为联系密切, 直接影响 着个体的选择、情绪、策略、耐性等多个面向 ${ }^{[9]}$ 。融 合 Amabile 的创造力理论, Tierney 与 Farmer ${ }^{[16]}$ 提出 “创新自我效能感”, 为创新行为的研究开拓了个体认 知层面的新视角。有研究表明, 领导者的行为能够对 员工的内在感知、努力程度和效能预期等多个方面产 生直接影响, 是创新自我效能感的重要前因变量 ${ }^{[18,19] 。}$ 分享型领导则是通过利益分享和权力分享两种途径 表示对员工创新的支持态度, 利益分享可以看作一种 “外在奖赏”, 外在奖赏是指工作之外的成果, 通常针 对员工的创新行为 ${ }^{[20]}$ 。外在奖赏作为一种激励源, 能 够弥补员工创新活动消耗的时间、精力和认知投入, 增加员工的获得感, 有利于员工积极配合领导者的创 新思路和方案, 促进共同的创新行为。分享型领导可 以基于合理的授权行为，给予下属员工的决策参与权 限, 能够使得其具有建言的空间和施展才华的更多机 会, 决策分享能够提升员工的尊重感知和组织支持感, 提升其面对创新困难的信心。同时, 员工能够在参与 决策的同时积极分享自身的知识 ${ }^{[14]}$, 促进团队成员间 
的信息沟通，有助于产生具有创意的想法。分享型领 导适当授权能够激发员工创新主动性, 使得员工在内 在创新驱动的条件下, 有信心持续努力的完成创新任 务 ${ }^{[14]}$ 。因此我们提出研究假设 $\mathrm{H} 2$ :

$\mathrm{H} 2$ : 分享型领导对创新自我效能感具有正向影 响。

在对于环境、人和行为的交互构面中，自我效能 感作为一种认知变量, 最适当描述人的动机、情感和 行动 ${ }^{[22]}$ 。Ford 认为自我效能感是能够激发个体创造行 为的一个关键动机因素 ${ }^{[23]}$ 。Luthans 认为具有高自我 效能感的员工能够积极调动各种资源, 促进工作任务 顺利完成 ${ }^{[24]}$ 。基于创新的内在动机理论, 创新信念对 于创新过程有极大的重要性, 创新过程充满了困难和 挑战, 还面临失败的风险, 拥有高创自我效能感的员 工, 才能不畏艰难, 持续保持积极的创新意愿, 为创 新活动付出努力 ${ }^{[24]}$ 。因此我们提出研究假设 $\mathrm{H} 3$ : 影响。

$\mathrm{H} 3$ ：创新自我效能感对员工创新行为具有正向

在众多关于环境变量 (如组织创新氛围、领导力 等) 和创新行为 (创造力) 的研究文献当中, 创新自 我效能感的中介作用被广泛研究, 可见其重要性, 员 工对于组织氛围、领导力的感知都能调节其创新自我

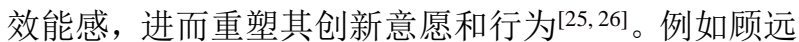
东和彭纪生验证了创新自我效能感能够介导组织创 新氛围与员工创新行为的关系 ${ }^{[9]}$ 。李朔等在创业型领 导对员工创新行为影响的研究中, 证实了创新自我效 能感的中介作用 ${ }^{[19]}$ 。分享型领导能够兼顾下属员工的 个人利益诉求和创新的内在动机, 同时给与创新员工 的外在激励和内在支持, 给与员工的管理授权和心理 授权 ${ }^{[27]}$, 有助于减少员工创新顾虑, 激发员工创新意 愿, 鼓舞其实现创新目标的努力, 能够积极影响员工 创新行为。由此可见, 创新自我效能感可能在分享型 领导与员工创新行为之间起着重要的媒介作用。基于 以上分析,我们提出假设 $\mathrm{H} 4$ :

H4：创新自我效能感在分享型领导与员工创新 行为之间起中介作用。

\section{3. 情感承诺的调节作用}

组织承诺是员工对于组织价值的认同度, 反映出 员工的情感归属程度 ${ }^{[28]}$ 。Meyer 等学者认为组织承诺 具备 3 个维度: 情感承诺、持续承诺和规范承诺, 分 别反映情感依附性、离开组织的成本对于个人行为的 约束性和感知到的个人责任感 ${ }^{[29]}$ 。三个维度中的情感 承诺最为核心, 重点体现了员工对于组织的认可度、 忠诚度和责任感。基于社会认同理论和自我决定理论, 当员工对于组织的认同度提升时, 才能具备良好的 “主人翁”意识, 自动的将组织的目标和自身的想法和 行动趋于同步, 将个人的才智积极投入到组织目标的 实现中 ${ }^{[30]}$ 。当员工处于高情感承诺时, 具备高认同、 高责任感的员工将会更加理解分享型领导的利益分
享行为和权力分享行为, 积极构建领导-员工的良好 交互关系，更加考虑组织和团队其他成员的共同利益， 愿意知识分享，体现良好的组织公民行为 ${ }^{[31,32]}$, 提出 有益于组织创新的想法, 并自愿的执行创新行为 ${ }^{[33]}$ 。

结合上文的论述，分享型领导能够提升员工的创 新自我效能感, 进而影响员工创新行为。由于处于高 情感承诺的员工, 在高自主动机的驱使下, 会积极配 合分享型领导的创新思路和举措, 有利于增加其面对 特定创新任务时的信心和抗风险韧性, 将创新想法全 力的落实下去。与之相反, 处于低情感承诺的员工, 对于组织的利益可能关心度不够, 也不愿意尽力配合 分享型领导的创新思路, 甚至表现冷漠, 不愿意进行 成员间的知识分享和沟通 ${ }^{[34]}$, 不利于创新想法的产生 和执行。据此本文可以推测:

$\mathrm{H} 5$ : 情感承诺正向调节分享型领导对员工创新行 为的影响。情感承诺程度越高, 分享型领导对员工创 新行为的影响越大; 情感承诺程度越低, 分享型领导 对员工创新行为的影响越小。

$\mathrm{H} 6$ :情感承诺对分享型领导与员工创新行为的正 向调节作用通过创新自我效能感中介作用得以发挥。

综上所述，构建的研究框架如图 1 所示:

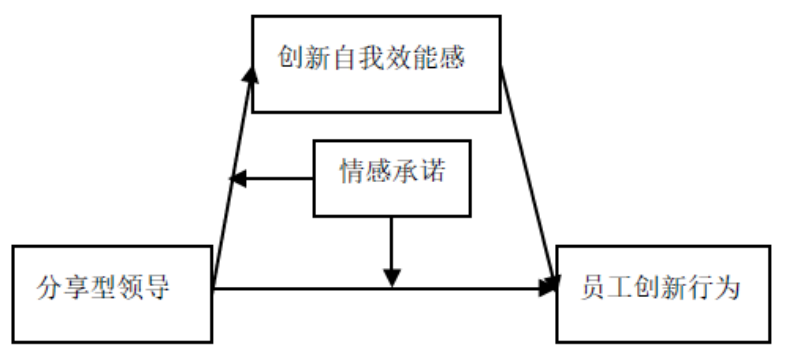

图 1 研究框架图

\section{3.研究方法}

\section{1. 数据来源}

本次调研通过问卷调研方式对研究数据进行收 集。调查对象为江苏、浙江等地的高新技术企业, 样 本根据《高新技术企业认定管理办法》（2018 年）进 行选取, 样本企业涉及电子信息、生物医药、机械制 造等多个技术领域。问卷对象以这些企业中研发部门 研发人员为主，包含部分企业管理人员。通过校友网 络、MBA 学员的关系网络, 进行问卷的发放和回收。 此次调研共发放问卷 300 份, 回收 220 份, 通过剔除 缺漏严重、答案规律性的问卷 32 份, 最终得到有效 问卷 188 份, 有效回收率为 $62.67 \%$ 。

\section{2. 变量测量}

本次调研在充分借鉴国内外被许多学者多次证 实的成熟量表, 它们具备良好的信度、效度。通过 Likert 五点量表进行测量, 1 表示“完全不同意”, 5 则 
表示“非常同意”。

分享型领导。基于龙立荣和陈琇霖 ${ }^{[34]}$ 开发的分享 型领导量表, 从名利分享和权力分享两个维度对分享 型领导进行度量, 共 10 个题项, 如“领导经常将归属 自己的荣誉让给下属”, “领导将自己的部分收入作为 奖金分给下属”, “领导经常和下属沟通交流，一起做 决策”等。Cronbach's $\alpha$ 值为 0.919 , 量表信度良好。

员工创新行为。主要采用 Scott 和 Bruce ${ }^{[35]}$ 所编 制的测量量表, 共 6 个题项, 如“工作中, 我会尝试运 用新的技术与方法”等。Cronbach's $\alpha$ 值为 0.827 , 量 表信度良好。

创新自我效能感。主要采用 Tierney 和 Farmer ${ }^{[16]}$ 编制的测量量表, 包含 3 个题项, 如“我有解决问题 的创造性方法”等。Cronbach's $\alpha$ 值为 0.842 , 量表信 度良好。

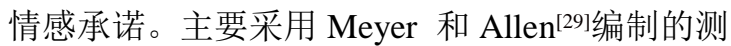
量量表, 共包含 6 个题项, 如“我对这个企业有强烈 的归属感”等。Cronbach's $\alpha$ 值为 0.860 , 量表信度良 好。

此外, 参照对于员工创新行为研究文献, 将年龄、 性别、受教育程度、工作年限和职级作为控制变量。

\section{4.数据分析与结果}

\section{1. 同源方差检验}

同源方差是指由于研究中采用了共同方法, 而造 成的一种系统性的偏差。由于本文数据均是由问卷调 查的方式获得, 可能存在同源方差的干扰。对题项进 行 Harman 单因素检验, 采用 Spss22.0 软件对所有核 心变量进行因子分析, 在未旋转的条件下, 析出 4 个 特征值大于 1 的主成分。首个因子的方差解释量为 $29.568 \%$, 低于 $40 \%$, 所有题项的解释总变异量为 $72.450 \%$, 因此数据的同源方差处于可接受范围。

\section{2. 验证性因子分析}

本文继续采用 Amos 24.0 对不同因子模型进行了 验证性因子分析, 分析结果如表 1 所示。其中, 各指 标值均显示出四因子模型要优于其他因子模型 $(\chi 2 / \mathrm{df}=1.064<3 ; \quad \mathrm{GFI}=0.911>0.9 ; \quad \mathrm{CFI}=0.992>0.9$; RSMEA $=0.018<0.08$ ), 表明四因子模型在统计学意义 上是显著的, 所以各量表区分效度良好。

表 1 验证性因子分析

\begin{tabular}{ccccc}
\hline 模型 & \multicolumn{1}{c}{$\chi^{2} / \mathrm{df}$} & GFI & CFI & RSMEA \\
\hline 模型 $\mathrm{a}$ & 1.064 & 0.911 & 0.992 & 0.018 \\
模型 b & 2.715 & 0.680 & 0.785 & 0.096 \\
模型 c & 2.702 & 0.672 & 0.777 & 0.095 \\
模型 $\mathrm{d}$ & 6.923 & 0.457 & 0.485 & 0.178 \\
\hline 注: FXLD: 分享型领导; CXXN: 创新自我效能感; QGCN: 情感承诺; YGCX: \\
员工创新行为。 a: FXLD, CXXN, QGCN, YGCX; b: FXLD+CXXN, QGCN, \\
YGCX; c: FXLD+CXXN, QGCN+YGCX; d: FXLD+CXXN +QGCN+YGCX。
\end{tabular}

\section{3. 相关性分析}

通过变量间的相关性分析可以对变量间关系进 行预判, 通过 Spss22.0 对各变量相关性分析的结果如 表 2 所示。可见主要的变量间均具有一定程度的相关 性, 初步支持了本文所提假设, 因此可以进行后续假 设检验。

表 2 描述性统计和相关性分析

\begin{tabular}{cccccc}
\hline 变量 & MEAN & SD & 1 & 2 & 3 \\
\hline 1.分享型领导 & 3.343 & 0.586 & & & \\
2.创新自我效能感 & 3.181 & 0.443 & $0.601^{* *}$ & & \\
3.情感承诺 & 3.597 & 0.532 & $0.238^{* *}$ & $0.439^{* *}$ & \\
4.员工创新行为 & 3.189 & 0.487 & $0.665^{* *}$ & $0.678^{* *}$ & $0.584^{* *}$ \\
\hline
\end{tabular}

\section{4. 层次回归分析}

研究采用分层线性回归的方法对所提假设进行 实证检验。对于中介效应的检验, 将参照 Baron 和 Kenny 提出的程序进行 ${ }^{[36]}$, 回归所得结果如表 3 所示 (见最后页)。M6 中分享型领导对于员工创新行为的 回归系数为正且显著 $(\beta=0.508, p<0.001)$, 表明分享 型领导能够显著促进员工创新行为, 即领导的利益分 享和权力分享行为越多, 员工越能够产生创新行为, $\mathrm{H} 1$ 得到支持。 M2 表明, 分享型领导显著促进创新自 我效能感 ( $\beta=0.427, p<0.001)$, 即领导越愿意分享, 员工对于完成创新任务的信心越强, H2 得证。M7 模 型显示, 创新自我效能感对于员工创新行为具有显著 正向影响 $(\beta=0.691, p<0.001)$, 表明创新自我效能 感能够显著促进员工创新行为, $\mathrm{H} 3$ 得到支持。 $\mathrm{M} 9$ 在 M6 的基础上加入了创新自我效能感 (中介变量), M9 与 $M 6$ 相比, 模型的 $R^{2}$ 值明显变化, 并且在 $M 9$ 中, 自变量分享型领导与中介变量创新自我效能感的系 数均显著, 说明加入中介变量是有意义的。加入中介 变量后, 分享型领导的系数由 $(\beta=0.508, p<0.001)$ 变为了 $(\beta=0.321, p<0.001)$, 因此, 创新自我效能 感在分享型领导与员工创新行为之间中起着部分中 介作用, H4 得证。

在验证情感承诺在假设模型中的调节效应之前, 首先应避免变量间的共线性问题, 研究对于分享型领 导和情感承诺进行了中心化处理, 对处理后的变量进 行乘积得到分享型领导和情感承诺的交互项。由表 3 中的 M4 可知, 分享型领导和情感承诺的交互项正向 调节分享型领导对创新自我效能的影响, 交互项系数 $(\beta=0.164, p<0.01)$, 在 M6 的基础上添加调节变量 和交互项得到模型 M8, 分享型领导和情感承诺的交 互项显著 $(\beta=0.178, p<0.01)$ 正向调节分享型领导 对员工创新行为的影响, H5 得证, 由 M10 可知, 组 织情感承诺对分享型领导与员工创新行为的调节作 用能够借由创新自我效能感得以发挥, 交互项系数 ( $\beta$ $=0.139, p<0.05), \mathrm{H} 6$ 得证。 
表 3 层级回归分析结果

\begin{tabular}{|c|c|c|c|c|c|c|c|c|c|c|}
\hline \multirow[t]{2}{*}{ 变量 } & \multicolumn{4}{|c|}{ 创新自我效能感 } & \multicolumn{6}{|c|}{ 员工创新行为 } \\
\hline & M1 & M2 & M3 & M4 & M5 & M6 & M7 & M8 & M9 & M10 \\
\hline 性别 & -0.091 & -0.086 & -0.093 & -0.072 & -0.066 & -0.06 & -0.003 & -0.049 & -0.022 & -0.032 \\
\hline 年龄 & $-0.138 * * * *$ & $-0.084 * *$ & $-0.058^{*}$ & -0.053 & $-0.193^{* * * *}$ & $-0.129 * * *$ & $-0.097 * * * *$ & $-0.082^{* * *}$ & $-0.092 * *$ & $-0.069 * *$ \\
\hline 受教育程度 & -0.010 & 0.003 & 0.001 & 0.006 & -0.013 & 0.003 & -0.006 & 0.004 & 0.001 & 0.002 \\
\hline 工作年限 & 0.025 & 0.003 & 0.002 & -0.001 & 0.049 & 0.023 & 0.031 & 0.017 & 0.021 & 0.017 \\
\hline 职级 & -0.055 & -0.046 & -0.045 & -0.046 & 0.006 & 0.018 & 0.045 & 0.018 & 0.038 & 0.029 \\
\hline 分享型领导 & & $0.427 * * *$ & $0.382 * * *$ & $0.407 * * *$ & & $0.508 * * *$ & & $0.464 * * * *$ & $0.321 * * *$ & $0.367^{* * * *}$ \\
\hline 情感承诺 & & & $0.247 * * *$ & $0.264 * * *$ & & & & $0.405 * * *$ & & $0.342 * * * *$ \\
\hline $\begin{array}{l}\text { 分享型领导* } \\
\text { 情感承诺 }\end{array}$ & & & & $0.164 * *$ & & & & $0.178^{* *}$ & & $0.139^{*}$ \\
\hline $\begin{array}{l}\text { 创新自我效 } \\
\text { 能感 }\end{array}$ & & & & & & & $0.691^{* * * *}$ & & $0.438^{* * * *}$ & $0.238^{* * * *}$ \\
\hline$R^{2}$ & $0.098^{* * *}$ & $0.402 * * *$ & $0.482 * * *$ & $0.497 *$ & $0.149 * * *$ & $0.506 * * *$ & $0.506 * * *$ & $0.684 * * *$ & $0.601 * * *$ & $0.707 * * *$ \\
\hline $\mathrm{F}$ & $3.955^{* * *}$ & $20.257 * * * *$ & $23.886^{* * * *}$ & $22.134 * * * *$ & $6.381 * * * *$ & $30.874 * * *$ & $30.946^{* * * * *}$ & $48.321^{* * * *}$ & $38.714 * * * *$ & $\begin{array}{c}47.769 * * \\
*\end{array}$ \\
\hline $\mathrm{R}^{2}$ 变化 & - & 0.304 & 0.08 & 0.016 & - & 0.357 & 0.357 & 0.178 & 0.095 & 0.106 \\
\hline
\end{tabular}

\section{5.研究结论与启示}

\section{1. 研究结论}

通过对于来自 188 份收集来自江苏、浙江等地高 新技术企业的调查数据进行分层回归, 实证分析了分 享型领导对于员工创新行为的影响, 同时检验了创新 自我效能感的中介作用和情感承诺的调节作用。研究 得出以下重要结论:

(1) 分享型领导对于员工创新行为具有显著的 正向作用。研究结果表明, 分享型领导能够显著促进 员工创新行为, 这与徐振亭等 ${ }^{[2]}$ 对于自我牺牲型领导 对员工创新行为具有显著正向影响的研究结果具有 相似性。同时, 与王宏蕾等 ${ }^{[37]}$ 对于授权型领导与员 工创新行为之间存在显著正相关关系的研究结果具 有相似性。

（2）创新自我效能感介导分享型领导与员工创 新行为的关系。分享型领导通过创新自我效能感显著 促进员工创新行为; 分享型领导对于提升员工的创新 自我效能感具有积极意义。分享型领导兼顾了利益分 享和决策分享, 能够在物质激励, 管理授权、心理授 权多个面向对于创新员工产生积极的影响, 提升其对 于完成特定创新任务的信心和持续性。本研究揭开了 分享型领导影响员工创新行为的“黑箱”, 使得对于员 工创新行为的前因变量研究更加丰富, 也为分享型领 导后续的相关研究开拓了思路。

（3）情感承诺正向调节分享型领导、创新自我效 能感及员工创新行为之间的关系。情感承诺作为个体 对于组织的价值认同度的衡量指标, 对于员工创新行 为以及领导-员工关系都有着重要的影响。实证结果
表明, 具有高情感承诺的员工, 更有利于提升员工创 新自我效能感在分享型领导对于员工创新行为影响 的中介作用。

\section{2. 管理启示}

本文实证结果对于企业中创新创业、构建和谐的 领导一员工关系实践具有一定程度的指导意义。

(1) 领导层面上, 通过研究发现, 分享型领导能 够通过利益分享和决策分享提升员工的创新行为。意 味着领导需要根据员工的多样性和差异性, 不断的提 升自身素养，根据不同的创新任务适当的调整领导风 格, 能够基于社会交换理论和员工自我角色认同理论, 在物质和心理两个方面, 构建一种和谐、有序的团队 氛围，不断支持员工、鼓励试错、大胆创新并向失败 学习的良好创新环境。提升员工的价值感、组织认同 感和自驱力, 增加员工的幸福感, 使得创新的整个过 程充满关怀和积极性, 实现创新的目标。

(2) 员工层面上,对于自身的角色认同应当有清 晰的认识, 在团队协作的过程中发掘自身对于整个团 队的价值, 树立起主人翁意识, 能够积极的与领导协 同, 与其他成员沟通交流, 集思广益, 群策群力, 提 升创新自我效能感，能够为创新目标的实现投入时间、 精力和才智, 将创新想法落实落地, 实现集体目标的 同时, 实现自身的全面提升, 获得工作的幸福感。

\section{3. 局限和研究展望}

研究进一步拓展了分享型领导与员工创新行为 的研究, 但是仍然存在一定的局限性, 未来需要进一 步的完善: 
（1）在对于中介效应的探讨当中, 仅仅考虑了员 工创新自我效能感这个变量, 分享型领导对于员工创 新行为的影响关系中, 可能存在其他的重要中介变量, 如工作重塑等值得深入研究, 同时, 未来可以进一步 拓展分享型领导的其他后果变量研究。

(2) 研究所用的数据本身是截面数据, 如果研究 变量间的动态关系时, 现有研究结果可能存在偏差, 未来研究可长期追踪关键变量的数据, 同时将多种类 型的企业纳入样本范围, 以便扩大研究结果的普适性。

\section{项目基金}

本文为教育部人文社会科学研究规划基金项目 《动态和竞争环境下双元创新对高新技术企业可持 续发展的影响机制研究》(18YJA630085) 的阶段性成 果之一。

\section{REFERENCES}

[1] Zhong Xi, Wang Tian, Fu Ye. (2020) Research on the relationship between insider identity perception and employee knowledge sharing[J]. Scientific Research Management, 41(7):181-188.

[2] Xu Zhenting, Luo Jinlian, Qu Yiying. (2020) Selfsacrificing leadership and employee innovation behavior: the cross-level effect of creative process input and team trust $[\mathrm{J}]$. Management Review, 32(11): 184-195.

[3] He Xiaobin, Zhu Danyang, Gao Yongqiang. (2020) The two dimensions of shared leadership and corporate innovation performance[J]. Studies in Science of Science, 38(4):747-758.

[4] Long Lirong, Chen Xiulin. (2021) Research on the Influence and Mechanism of Shared Leadership on Employees' Perception of Organizational Harmony[J]. Chinese Journal of Management, 18(2):213-222.

[5] Qin Dajia, Li Genyi, Shi Yi, et al. (2020) Research on the Mechanism of the Perceived Career Sustainability of Grassroots Skilled Employees on Their Innovative Behaviors_-Based on the Perception of Yin-Yang Harmony[J]. Management Review, 32(9) :205-219.

[6] Long Lirong, He Wei, Chen Xiulin. (2016) Leadership from willingness to success[J]. Tsinghua Management Review, (3): 54-59.

[7] Ding He, Lin Xinqi, Xu Yangyang. (2018) Research on the Influence Mechanism of Advantage- $b$ ased Psychological Atmosphere on Innovation Behavior[J]. Nankai Management Review, 21(1):28-38.

[8] Wang Yongyue, Wang Huijuan, Wang Xiaochen. (2015) The influence of insider's identity perception on employees' innovative behavior: the role of innovative self-efficacy and authority compliance[J]. Psychological Science,38(4):954959.

[9] Gu Yuandong, Peng Jisheng. (2010) The influence of organizational innovation atmosphere on employee innovation behavior: the mediating role of innovation self-efficacy[J]. Nankai Management Review, 13(1): 30-41.

[10] Huang Gui. (2010) Why can't companies that emphasize "dedication" get what they want? Based on the thinking of the exchange relationship between state-owned enterprise organizations and employees[J]. Management World, (11):105-113.

[11] Huang Yong, Yang Jie, Hu Saisai. (2020) Organizational support and employee creativity: the influence of relative organizational support and emotional commitment [J]. Journal of Guizhou University of Finance and Economics, (5): 80-87.

[12] Wang Wei, Yu Jiping, Zhang Shanliang. (2019) The influence mechanism of empowered leadership on employee tacit knowledge sharing: the mediating role of insider identity perception and the moderating role of workplace friendship [J]. Science and Technology Progress and Policy, 36(7):123-130.

[13] Xu Xixiong, Gan Weiyu. (2011) Organizational Support Fit, Psychological Empowerment and Employee Organizational Commitment: A Theoretical Framework of Employment Relationship Management of New Generation of Migrant Workers - A Case Study Based on Haidilao[J]. Management World, (12) :131-147.

[14] Liu Peiqi, Liu Bing, Li Chang. (2018) The influence of empowered leaders on knowledgebased employees' willingness to share knowledge: Based on the perspective of social information Technoeconomics,37(7):81-87.

[15] Gong Hong, Huo Yanhui. (2017) The relationship between shared leadership and organizational commitment in the communications industry: based on the mediating role of psychological empowerment[J]. Economic Research Guide, (6):164-167.

[16] Tierney P, Farmer S M. (2002) Creative SelfEfficacy: Its Potential Antecedents and Relationship to Creative Performance[J]. Academy of Management Journal, 45(6):11371148. 
[17] Yang Jingzhao, Yang Dongtao, Zhao Shundi, etc. (2011) "I am", "I can", "I wish"-the relationship between employee innovation psychological factors and employee innovation $[\mathrm{J}]$. Science of Science and Management of Science and Technology, 32(4):165-172.

[18] Jia Jinan, Kong Xiangxue, Wang Shenyue. (2020) Research on the influence of error management atmosphere on the innovative behavior of the new generation of employees[J]. Scientific Research Management,41(9):238-246.

[19] Li Shuo, Yi Lingfeng, Yin Yishuai. (2020) How can entrepreneurial leaders stimulate employees' innovative behavior? -On the role of innovative self-efficacy and organizational identification[J]. Hubei Social Sciences, (11):8390 .

[20] Huang Qiufeng, Tang Ningyu, Chen Zhijin, et al. (2017) Research on the impact of transformational leadership on employees' innovative behaviors: a meta-analysis test based on self-determination theory and social cognitive theory $[\mathrm{J}]$. Research and Development Management, 29 (4):73-80.

[21] Lu Jihua, Chen Lili, Zhao Xinan. (2013) Research on the relationship between organizational support, organizational commitment and knowledge-based employee engagement[J]. Science of Science and Management of Science and Technology, (1):147-153.

[22] Bandura A. (1977) Self-efficacy: toward a unifying theory of behavioral change.[J]. Advances in Behaviour Research \& Therapy,1(4):139-161.

[23] Ford C M. (1996) A Theory of Individual Creative Action in Multiple Social Domains[J]. The Academy of Management Review,21(4):11121142 .

[24] Stadjkovic A D, Luthans F, Slocum J W. (1998) Social cognitive theory and self-efficacy: Goin beyond traditional motivational and behavioral approaches $[\mathrm{J}]$. Organizational Dynamics, 26(4):62-74.

[25] Ma Wei, Su Hang. (2020) The impact of differential atmosphere perception on employee innovation behavior[J]. Science \& Technology Progress and Policy, 37(21): 136-143.

[26] Yan Liang, Zhang Zhihe. (2017) The mixed influence mechanism of organizational innovation atmosphere on employees' innovative behaviors[J]. Scientific Research Management, 38(9):97-105.
[27] Li Yongzhan. (2018) The impact of transformational leadership on employee innovation behavior: the role of psychological empowerment and emotional commitment [J]. Scientific Research Management, (7): 123-130.

[28] Fan Yun, Zhang Xu, Yan Jing. (2013) A review of organizational commitment research based on theoretical evolution [J]. Management Review, (1): 101-113.

[29] Allen N, Meyer J. (1990) The Measurement and Antecedents of Affective, Continuance and Normative Commitment to the Organization[J]. Journal of Occupational Psychology,63(1):1-18.

[30] Zhang Xu, Fan Yun, Huang Minping, et al. (2013) Construction of organizational commitment formation mechanism model based on selfdetermination theory: with autonomous demand becoming dominant demand as a background[J]. Nankai Management Review, (6): 59-69.

[31] LYU Fuxin, Gu Shanshan. (2007) Analysis of the correlation between psychological ownership and organizational citizenship behavior: Based on the perspective of local enterprises and the empirical evidence of Zhejiang enterprises[J]. Management World, (5):94-103.

[32] Zhao Hongjian, Bai Shaojun, Cui Mengxiao. (2019) The construction of high-performance work system and organizational citizenship behavior model: the dual mediating effect of PIED and emotional commitment[J]. Science and Technology Progress and Policy, (17):136-141.

[33] Liu Xiaoping. (2011) The formation process of employee organizational commitment: internal mechanism and external influence-An empirical study based on social exchange theory [J]. Management World, (11): 92-104.

[34] Yang Fu, Zhang Lihua. (2012) The influence of team communication and work insecure atmosphere on innovative behavior: the moderating effect of creativity self-efficacy[J]. Bulletin of Psychology, 44(10): 1383-1401.

[35] Scott S G, Bruce R A. (1994) Determinants of Innovative Behavior: A Path Model of Individual Innovation in the Workplace $[\mathrm{J}]$. Academy of Management Journal,37(3):580-607.

[36] Baron R M, Kenny D A. (1999) The moderatormediator variable distinction in social psychological research: conceptual, strategic, and statistical considerations. [J]. J Pers Soc Psychol,51(6):1173.

[37] Wang Honglei, Sun Jianmin. (2018) Authorized leadership and employee innovation behavior: the moderating effect of structural formalization[J]. Management Science,31(3):29-39. 